\title{
Chaos Glial Network Connected to Multi-Layer Perceptron for Solving Two-Spiral Problem
}

\author{
Chihiro Ikuta \\ Dept. of Electrical and Electronic Eng., \\ Tokushima University \\ 2-1 Minami-Josanjima, Tokushima, Japan \\ E-mail: ikuta@ee.tokushima-u.ac.jp
}

\author{
Yoko Uwate \\ University / ETH Zurich, \\ Winterthurerstrasse 190, \\ CH-8057 Zurich, Switzerland, \\ Email: yu001@ini.phys.ethz.ch
}

\author{
Yoshifumi Nishio \\ Dept. of Electrical and Electronic Eng., \\ Tokushima University \\ 2-1 Minami-Josanjima, Tokushima, Japan \\ E-mail: nishio@ee.tokushima-u.ac.jp
}

\begin{abstract}
Some methods using artificial neural network were proposed for solving to the Two-Spiral Problem (TSP). TSP is a problem which classifies two spirals drawn on the plane, and it is famous as the high nonlinear problem.

In this paper, we propose a chaos glial network which connected to Multi-Layer Perceptron (MLP). The chaos glial network is inspired by astrocyte which is glial cell in the brain. By computer simulations for solving TSP, we confirmed that the proposed chaos glial network connected to MLP gains better performance than the conventional MLP.
\end{abstract}

\section{INTRODUCTION}

Back Propagation (BP) was introduced by Rumelhart in 1986 [1]. BP is used for learning algorithm of MLP and the error propagates backwards in the network. MLP using BP algorithm is well known to perform for the pattern classification tasks. However, the solution of the network often falls into the local minimum, because BP uses the steepest descent method for the leaning process. Generally, if the solution of MLP falls into the local minimum, it can not escape. In order to avoid this problem, some methods to release the solution from the local minimum are required.

Recently, the mechanism of astrocyte which is glial cell existing in the central nervous system of the brain has been attracting. Several research groups discovered that astrocytes affect to neurons with signal transduction [2]. We consider that astrocytes make good effects to neurons in the biological neural networks.

In this study, we propose a chaos glial network which connects to MLP as shown in Fig. 1. We consider that glial cells produce chaotic oscillation which is affected to neurons. This view is motivated by investigations of the Hopfield network solving combinatorial optimization problems with the help of a chaotic input signal component, designed in order to avoid local minima. It appears, from computer simulations, that a chaotic input component may substantially enhance the capability of avoiding these local minima [3]-[5]. Hence, we believe that chaotic signals may be used to further enhance the efficiency of the proposed chaos glia neural network.

Furthermore, chaotic oscillation generated from glial cells propagates to the neighbor glial cells. Namely, certain neuron in this network is affected from some of glial cells located at a nearby site. We apply the proposed chaos glial network connected to MLP for solving TSP and confirm the efficiency by computer simulations.

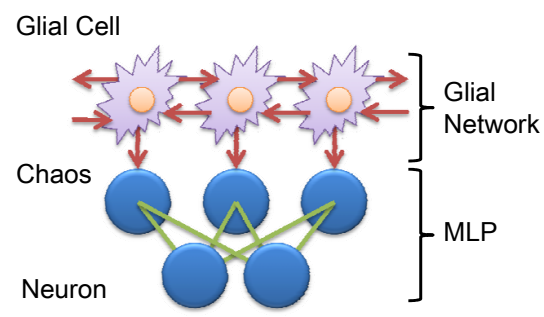

Fig. 1. Conceptual chaos glial network.

\section{Multi-Layer Perceptron}

MLP is a most famous feed forward neural network. This network is used for pattern recognition, pattern classification, and other tasks. MLP has some layers, it has mainly input layer, hidden layer, and output layer. Generally, it is known that MLP can solve a more difficult task if the number of layer or neuron is increased. We consider MLP which is composed of four layers (one input layer, two hidden layers and one output layer), and MLP has the chaos glial network in the second layer of the hidden layer. The proposed MLP with the chaos glial network structure (connected 2-20-40-1) is shown in Fig. 2.

\section{A. Neuron Updating Rule}

The updating rule of neurons in the input layer, the first hidden layer and the output layer is described by Eq. (1) which is conventional updating rule.

$$
x_{i}(t+1)=f\left(\sum_{j=1}^{n} w_{i j}(t) x_{j}(t)-\theta_{i}(t)\right),
$$

In the chaos glial neural network, chaotic oscillation is added to neurons in the second hidden layer. This neuron's 


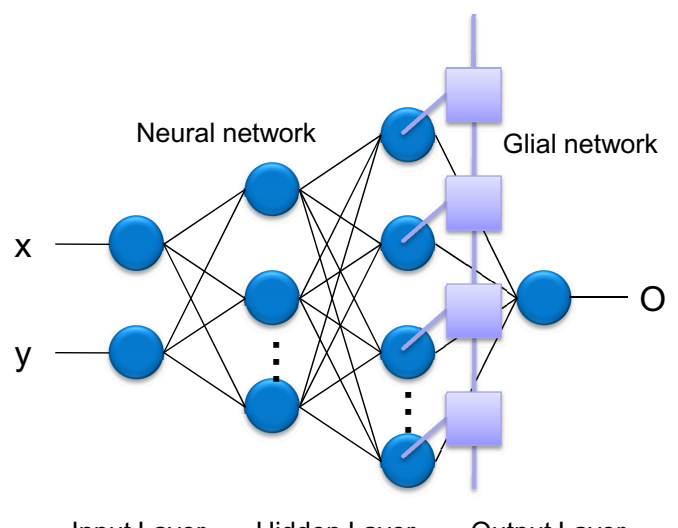

Input Layer Hidden Layer Output Layer

Fig. 2. Chaos glial network connected to MLP.

updating rule is following as Eq. (2).

$$
x_{i}(t+1)=f\left(\sum_{j=1}^{n} w_{i j}(t) x_{j}(t)-\theta_{i}(t)+\alpha \Psi_{i}(t)\right)
$$

where $x$ : input or output, $w:$ weight parameter, $\theta:$ threshold, $\psi, \Psi$ : chaotic oscillation, $\alpha$ : amplitude of chaos and $f$ : output function. And we use sigmoid for the output function as Eq. (3).

$$
f(a)=\frac{1}{1+e^{-a}}
$$

In the biological neural network, it is known that the glial cells affect to the neighbor neurons over a wide range by propagating in the network [7]. In order to realize this phenomena, we add chaotic oscillation to neurons by using Eq. (4).

$$
\Psi_{i}(t)=\sum_{k=-m}^{m} \beta^{|k|} \psi_{i+k}(t-|k|),
$$

where $\beta$ denotes attenuation parameter and $k$ is the propagating range in the glial network. Chaotic oscillation is propagating in the glial network as shown in Fig. 3 and it takes time depending on distance. For example, when the glial cell is located three units far from certain neuron, effect of chaotic oscillation arrives to the neuron after three learning steps. And chaotic oscillation decreases while chaos is propagating in the network.

\section{B. Back Propagation}

The error of MLP propagates backward in the feed forward neural network. BP algorithm changes value of weights to obtain smaller error than before. The error of the network is given by Eq. (5).

$$
E=\frac{1}{2} \sum_{i=1}^{n}\left(t_{i}-O_{i}\right)^{2},
$$

where E: error value, t: target value and $\mathrm{O}$ : neuron output. By changing the value of weights, MLP's error becomes smaller.

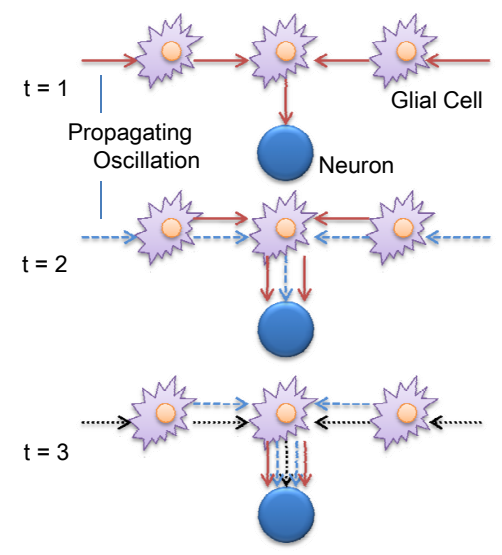

Fig. 3. Propagating chaos in the network.

Therefore, partial differential of the weight is carried out by Eq. (6).

$$
\Delta W_{k j}=-\eta \frac{\partial E}{\partial w_{k j}}
$$

\section{Chaotic Oscillation of Glial Network}

We use skew tent map to generate chaos. This map is one of simple chaotic maps and the center of map is shifted for a little from the standard tent map. This chaotic map is defined by Eq. (7) and the diagram of this map is shown in Fig. 4. Neurons in the second hidden layer are affected chaotic oscillation by Eq. (7) into the Eq. (4).

$$
\psi_{i}(t+1)=\left\{\begin{array}{ll}
\frac{2 \psi(t)+1-A}{1+A} & (-1 \leq \psi(t) \leq A) \\
\frac{-2 \psi(t)+1+A}{1-A} & (A<\psi(t) \leq 1)
\end{array},\right.
$$

Here, we consider the case that we prepare two similar initial

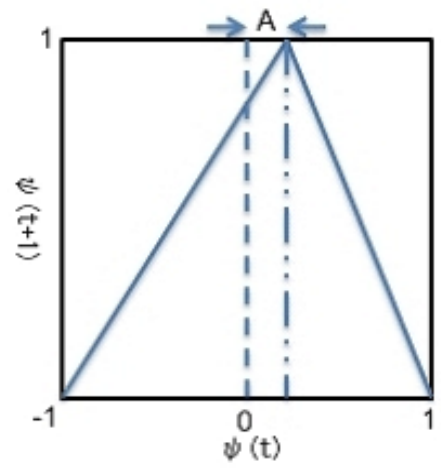

Fig. 4. Skew tent map $(A=0.05)$.

values of chaos map for adding two adjacent neurons. Figure 5 shows the two time series obtained from the skew tent map when initial values are fixed as $\psi_{2}(0)=0.12459$ and $\psi_{2}(0)=$ 0.12460 . From this figure, the plots of the two time series are spread and difference between two time series becomes large with time, even if we use similar initial value to generate 


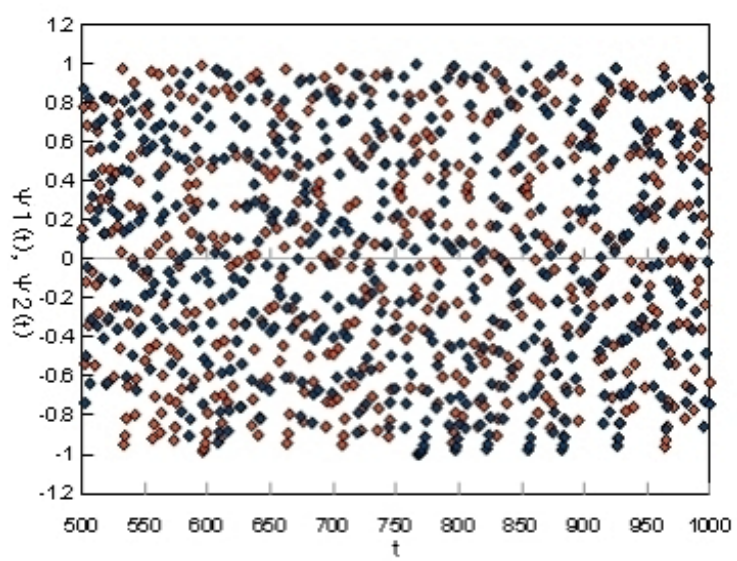

Fig. 5. Time series of skew tent map.

chaos. This is typical phenomena of chaos known as butterfly effect. If glial cells add these chaos to neurons, each neuron is affected with a completely different oscillation.

However, in the real biological neuro-glial network, glial cells affect each other with neighbor cells. We use the chaos glia propagating equation (Eq. 4) for having correlation each other of neighbor glial cells. Figure 6 shows two chaotic time series by using Eq. (4). The parameters are fixed as $\beta=0.8$, $m=5$. Two chaotic time series have similar peek points. We consider that these chaotic time series have some correlations.

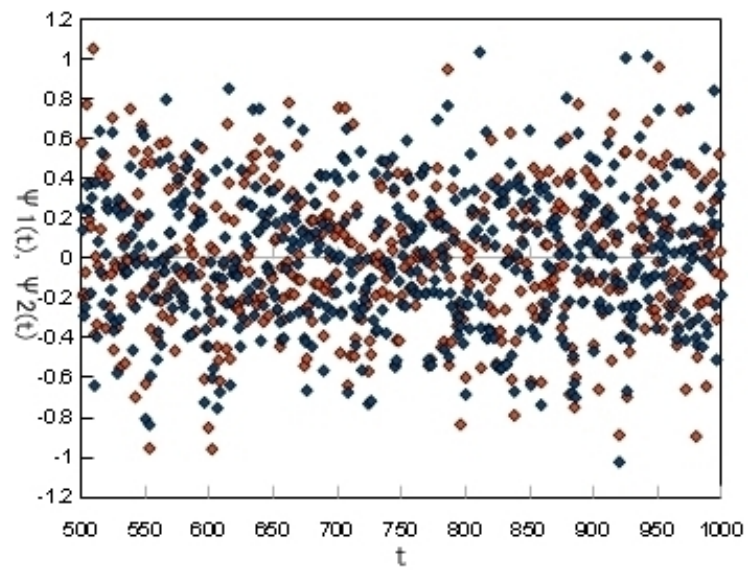

Fig. 6. Time series of skew tent map with correlation.

\section{Simulations}

In this section, the difference in the performance of our MLP; chaos glial network connected to MLP and the conventional MLP is compared.

\section{A. Two-Spiral Problem}

We apply the proposed network for solving TSP [6]. MLP learns to each point of two spirals, and MLP learns by using BP algorithm.

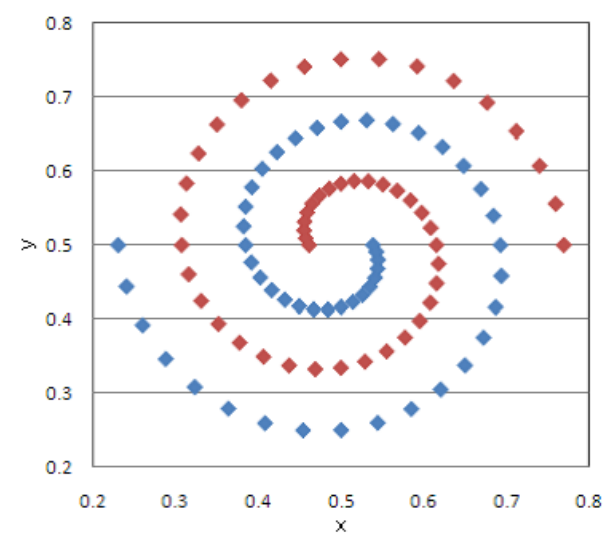

Fig. 7. Two-spiral problem.

\section{B. Simulation Result}

Each MLP learns the two spirals by setting up same weights before learning process. We prepare 98 data of two spirals as shown in Fig. 7. The number of learning points is fixed as 500000. We investigate the error which is modified in the meantime. The error function is defined as Eq. (8).

$$
E=\frac{1}{n} \sum_{i=1}^{n}\left|t_{i}-O_{i}\right|,
$$

where $E$ : error value $t:$ target value, $O$ : output value.

\section{Changing the number of neuron adding chaos}

We investigate the learning ability when the number of neuron adding chaos is changed. In this simulation, we add chaos to the neurons in the second hidden layer's. We change the percentage of neuron adding chaos as follows $0 \%, 20 \%$, $50 \%$, and $100 \%$. The average result for 30 times of simulations is summarized in Tab. I.

TABLE I

The Performance of MLP with Chaos Glial Network by Changing the Number of Neuron Adding Chaos

\begin{tabular}{|c||c|c|c|c|}
\hline Points & $0 \%$ & $20 \%$ & $50 \%$ & $100 \%$ \\
\hline \hline 100000 & 0.306195 & 0.334227 & 0.334600 & 0.322175 \\
\hline 150000 & 0.228353 & 0.269349 & 0.224780 & 0.244479 \\
\hline 200000 & 0.170263 & 0.198626 & 0.177127 & 0.166893 \\
\hline 250000 & 0.135390 & 0.153809 & 0.147403 & 0.143317 \\
\hline 300000 & 0.120928 & 0.140445 & 0.116600 & 0.115145 \\
\hline 350000 & 0.100454 & 0.136136 & 0.111645 & 0.103821 \\
\hline 400000 & 0.090179 & 0.120175 & 0.111659 & 0.081798 \\
\hline 450000 & 0.088718 & 0.121040 & 0.094112 & 0.086527 \\
\hline 500000 & 0.094303 & 0.109110 & 0.086106 & 0.074204 \\
\hline \hline Minimum & 0.018176 & 0.010911 & 0.002383 & 0.002523 \\
\hline
\end{tabular}

From Tab. I, we obtain the best learning ability when all neurons in the second hidden layer are added chaotic oscillation. In the next simulation, we add chaotic oscillation to every neurons in the second hidden layer. 


\section{Results of Each MLP Learning}

We compare the chaos glial network connected to MLP, the conventional MLP and the random glial network connected to MLP. In the random glial network, glial network produces random oscillation. Each result is summarized in Tab. II. This result shows the average result for 50 times of simulations.

TABLE II

The Performance By Using Each MLP

\begin{tabular}{|c||c|c|c|}
\hline Point & Conventional & Random Noise & Chaotic Noise \\
\hline \hline 100000 & 0.274507 & 0.299191 & 0.299990 \\
\hline 150000 & 0.189218 & 0.228571 & 0.247936 \\
\hline 200000 & 0.142849 & 0.165421 & 0.197222 \\
\hline 250000 & 0.111989 & 0.136988 & 0.152111 \\
\hline 300000 & 0.084422 & 0.118154 & 0.120912 \\
\hline 350000 & 0.093832 & 0.098092 & 0.097169 \\
\hline 400000 & 0.081747 & 0.086327 & 0.080341 \\
\hline 450000 & 0.079630 & 0.087916 & 0.074895 \\
\hline 500000 & 0.076502 & 0.083399 & 0.067850 \\
\hline \hline Minimum & 0.0125310 & 0.0104630 & 0.0072510 \\
\hline
\end{tabular}

At start learning, conventional MLP is better than the others. However, after 400000 learning points the chaos glial network connected to MLP gains better performance than the conventional MLP. The random glial network connected to MLP shows the most worst result. If we focus on the minimum error, the chaos glial network connected to MLP can find smallest error value. Because similarity of near neurons performs well by effects of chaos glial cells. And when MLP falls into local minima, chaos helps to escape with effectively.

Figure 8 is a typical example of learning curve. The random glial network connected to MLP can not learn to two spirals. The chaos glial network connected to MLP and the conventional MLP can learn to it, and the chaos glial network converges to the lower error value. The learning curve of the

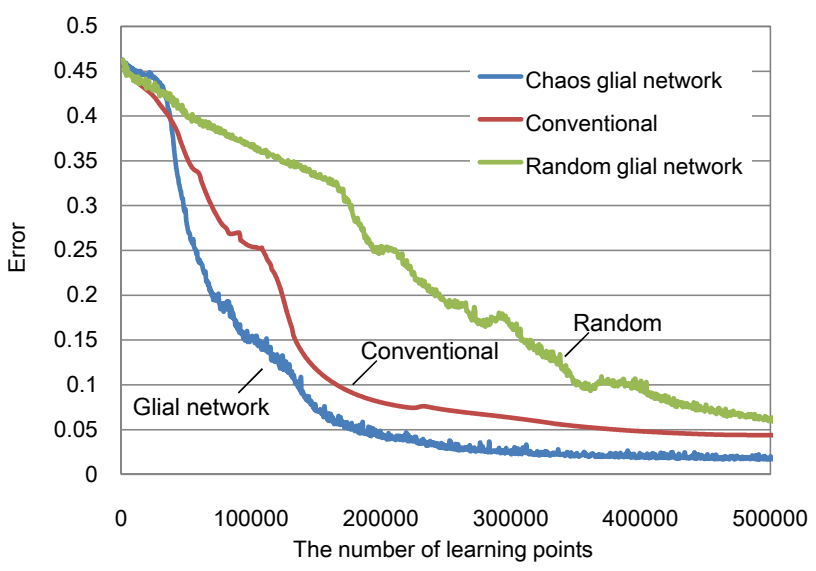

Fig. 8. The error curve by three MLP networks.

conventional MLP is smooth. While, the others have little vibration. We consider that this vibration phenomena makes to escape out from the local minimum.

\section{E. Classification of Two Spirals}

In this section, the chaos glial network connected to MLP classifies the two spirals. The network learns to two spiral points by propagating chaos method. After learning, we puts points $(0.2 \leq x \geq 0.8,0.2 \leq y \geq 0.8)$ changing little by little in MLP's input layer. Figure 9 show the simulation result. In this case, the error value is around 0.05 at 500000 learning points. When MLP's output is nearer 1, this point is classified to red spiral. And when MLP's output is nearer 0, this point is classified to blue spiral. This result shows that the chaos glial network connected to MLP can solve TSP.

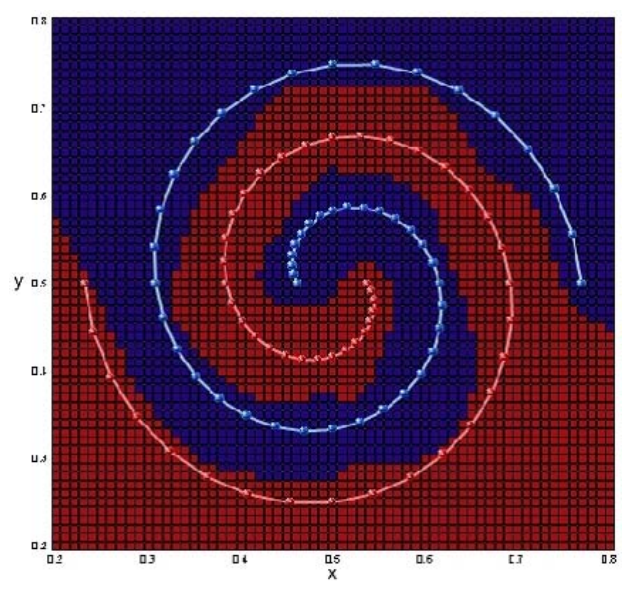

Fig. 9. Classification of two spirals.

\section{CONCLUSION}

In our study, we have proposed a chaos glial network. This network gave chaotic oscillations to the second hidden layer's neuron and this chaotic oscillation propagates to other neurons. We confirmed that the chaos glial network connected to MLP gains better performance than the conventional MLP and into the random glial network connected to MLP for solving TSP.

\section{REFERENCES}

[1] D.E. Rumelhart, G.E. Hinton, and R.J. Williams, "Learning representations by back-propagating errors," Nature, vol. 323-9, pp. 533-536, 1986.

[2] P.G. Haydon, "Glia: listening and talking to the synapse," Nature Reviews Neuroscience, vol 2, pp. 844-847, 2001.

[3] Y. Hayakawa, A. Marumoto, and Y. Sawada, "Effects of the chaotic noise on the performance of a neural network model for optimization problems," Physical Reviews E, vol. 51, no. 4, pp. 2693-2696, Apr. 1995.

[4] T. Ueta, Y. Nishio, and T. Kawabe, "Comparison between Chaotic Noise and Burst Noise on Solving Ability of Hopfield Neural Networks," Proc. NOLTA'97, vol. 1, pp. 409-412, Nov. 1997.

[5] Y. Uwate, Y. Nishio, T. Ueta, T. Kawabe, and T. Ikeguchi, "Performance of Chaos and Burst Noise Injected to the Hopfield NN for Quadratic Assignment Problems," IEICE Transactions on Fundamentals, vol. E87A, no. 4, pp. 937-943, Apr. 2004. 2004.

[6] J.R. Alvarez-Sanchez, "Injecting knowledge into the solution of the twospiral problem," Neural Computing \& Applications, vol. 8, pp. 265-272, 1999

[7] S. Koizumi, M. Tsuda, Y. Shigemoto-Nogami, and K. Inoue, "Dynamic inhibition of excitatory synaptic transmission by astrocyte-derived ATP in hippocampal cultures," Proc. National Academy Science of the U.S. A, vol. 100, pp. 11028-11028, 2003. 